\title{
Variación morfosintáctica y lenguas en contacto: las formas analíticas y sintéticas del presente progresivo en el español monolingüe y bilingüe
}

\author{
Stephen Fafulas y Manuel Diaz-Campos* \\ Universidad de Indiana, Estados Unidos
}

\begin{abstract}
Resumen
En este trabajo se realiza un análisis cuantitativo sobre la variación morfosintáctica de las formas sintéticas (presente simple) y analíticas (presente progresivo) en contextos cuyo significado alude al tiempo presente progresivo en el español monolingüe y bilingüe. Se emplea una encuesta contextualizada, previamente codificada según el aspecto léxico-semántico del verbo y el valor semántico de los adverbios de tiempo, con el objetivo de evidenciar los contextos en los que hay variación de dichas formas. Además de los factores lingüísticos, se incluyen los factores extralingüísticos de género y bilingüismo en el análisis. Los resultados señalan que tanto el aspecto léxico-semántico del verbo como el valor semántico del adverbio son factores significativos en la predicción de ambas formas. De igual manera, el factor extralingüístico que considera el nivel de bilingüismo de los participantes tiene un efecto significativo. El estudio contribuye metodológicamente a la investigación de la variación de las formas analíticas y sintéticas, dado que el instrumento empleado en la presente monografía controla las variables lingüísticas
\end{abstract}

\footnotetext{
*Para correspondencia, dirigirse a: Stephen Fafulas (sfafulas@indiana.edu), Department of Spanish and Portuguese, Indiana University, Ballantine Hall 857, 1020 E, Kirkwood Avenue, Bloomington, IN 47405-7103, USA.
} 
y obtiene el mismo número de respuestas por persona, permitiendo una evaluación de los participantes en contextos idénticos. Por último, el estudio aporta a la literatura previa sobre el tema al proveer evidencia de que las formas sintéticas y analíticas aparecen en alternancia en un número de contextos mayor que los previamente identificados en otros estudios, lo cual permite definir debidamente el ámbito de la variación.

Palabras clave: variación morfosintáctica, sociolingüística, aspecto léxico, contacto inglés-español, bilingüismo, español.

\author{
MORPHOSYNTACTIC VARIATION AND LANGUAGE CONTACT: \\ ANALYTIC AND SYNTHETIC FORMS OF THE PRESENT PROGRESSIVE IN \\ MONOLINGUAL AND BILINGUAL SPANISH
}

\begin{abstract}
In this study we present a quantitative analysis of the morphosyntactic variation of the analytic (present progressive) and synthetic (simple present) forms of present progressive aspect in monolingual and bilingual Spanish. By way of a contextualized questionnaire previously coded for the linguistic factors of verbal lexical aspect and semantics of the adverb, we identify the contexts of variation of the forms under investigation. In addition, the analysis includes the extralinguistic factors of gender and bilingualism. The results indicate that lexical aspect of the verb, as well as the semantic value of the adverb, are significant factors in the prediction of both forms. Similarly, the factor regarding participant level of bilingualism has a significant effect. We contribute methodologically to the study of the variation of the analytic and synthetic forms of Spanish present progressive aspect given that the instrument employed in the current study controls the linguistic variables and obtains the same number of responses per person, allowing for a comparable evaluation of participants in similar contexts. Finally, we add to the previous literature on this topic by providing evidence that the synthetic and analytic forms appear in variation in a larger number of contexts than previously recognized, allowing for an accurate definition of the variable context.
\end{abstract}

Key words: morphosyntactic variation, sociolinguistics, lexical aspect, contact English-Spanish, bilingualism, Spanish.

Recibido: 28/05/10. Aceptado: 09/09/10. 


\section{INTRODUCCIÓN}

Como en otras lenguas romances, el español cuenta con dos formas verbales para indicar la noción semántica de "acción en curso" (aspecto) en el tiempo presente: (1) la forma sintética (presente simple), "Mira, empieza el partido ahora", y (2) la forma analítica (presente progresivo), "Mira, está empezando el partido ahora". Sin embargo, hasta qué punto estas formas son intercambiables y en qué contextos específicos se observa variación es algo que requiere ser determinado. Las monografías previas sobre el presente progresivo en español (Cortés-Torres 2005; Klein 1980; Koontz-Garboden 2004; Márquez Martínez 2009; Sánchez-Muñoz 2004) no presentan resultados comparables en cuanto a la variación de las formas analíticas y sintéticas, dado que estos no se enfocan en hablantes monolingües del español sino que apuntan a la transferencia del inglés en hablantes bilingües en contextos donde el inglés está en contacto con el español. Por lo tanto, las preguntas centrales que motivan el presente estudio son las siguientes: ¿Qué factores lingüísticos y extralingüísticos influyen en la selección de las formas analíticas y sintéticas del presente progresivo? ¿Hasta qué punto existen contextos que propician variación en los hablantes monolingües del español y los bilingües cuya lengua materna es el español?

Estudios previos (Butt y Benjamin 1994; Bybee et al. 1994; Comrie 1976; Gili-Gaya 1964), que analizan el presente progresivo en español e inglés, indican que el español se diferencia del inglés básicamente en que en español existen dos formas que expresan la noción semántica de "acción en progreso", mientras que en inglés solamente hay una (mediante la estructura be + -ing). Por lo tanto, los estudios empíricos sobre este tema (Klein 1980; Koontz-Garboden 2004; Márquez Martínez 2009; Sánchez-Muñoz 2004; Torres Cacoullos 2000) proponen la transferencia como factor principal para explicar la variación entre las formas sintéticas y analíticas en los hablantes bilingües. El problema es que mientras sugieren que hay variación entre estas dos formas en español, no proveen datos concretos acerca de todos los contextos posibles analizados exhaustivamente para respaldar su suposición. Es importante destacar que estos estudios no analizan el comportamiento lingüístico de hablantes monolingües del español que no tienen contacto con el inglés. Los hablantes clasificados como monolingües en estos estudios - de hecho- tienen contacto con el inglés, dado que viven en los Estados Unidos. Otra limitación de estos estudios es que no son coherentes en los contextos lingüísticos que se identifican para comparar a los bilingües frente a los monolingües y, por lo tanto, no es nada sorprendente que sus resultados sean inconsistentes. Como ya es sabido en los estudios 
de variación sociolingüística, hay que identificar los contextos en los que dos o más formas se neutralizan para poder hablar de la variación de dichas formas (Labov 1972; Sankoff 1988; Silva-Corvalán 2001). La metodología de nuestro estudio intenta ser más precisa que los estudios ya mencionados en la identificación de los contextos en que las formas sintéticas y analíticas del presente progresivo se producen y, a la vez, se controlan las variables lingüísticas de una manera que sirve para determinar el peso que tienen los factores lingüísticos en la selección de estas formas.

\section{ANTECEDENTES}

\subsection{Aspecto LÉXico y gramatical}

En el presente estudio examinamos el papel del aspecto léxico del verbo para medir su peso como factor determinante en la selección de la forma sintética o analítica en el español monolingüe y bilingüe. Otros estudios de tiempo-aspecto han demostrado que las propiedades semánticas de las clases aspectuales de verbos (logros, realizaciones, actividades y estados) tienen una influencia importante en el vínculo de estas formas verbales con una morfología específica (Bardovi-Harlig 2000; Bosque y Demonte 1999; Vendler 1967). El presente estudio toma estos antecedentes como elementos de base para presentar una revisión básica acerca de la noción de aspecto.

El aspecto se diferencia del tiempo, que ubica un evento según su cronología, por su referencia al componente temporal inherente en un verbo o predicado (Comrie 1976). El aspecto léxico (tabla 1) se refiere, básicamente, a si un verbo muestra una acción durativa, como caminar, si es puntual como llegar, o bien si muestra elementos de los dos en un punto de culminación como el verbo cantar en la oración; cantar una canción.

Tabla 1. Ejemplos de verbos y de las clases aspectuales

Estados (EST): saber, creer, querer.

Actividades (ACT): caminar, cantar, jugar.

Realizaciones (REA): cantar una canción, caminar $5 \mathrm{~km}$.

Logros (LOG): despertarse, llegar. 
Ejemplificamos estos puntos en (1). José corre (presente) y José corrió (pretérito) en (1a) presentan una diferencia en la cronología del tiempo. Por otro lado, (1b) (José corría (imperfecto) y José estaba corriendo (pretérito progresivo)) ilustra una diferencia de aspecto gramatical. Es importante para nuestros propósitos notar que mientras en el ejemplo (1b) hay un contraste respecto del aspecto gramatical, el aspecto inherente del verbo no cambia.

(1) a. Tiempo

b. Aspecto gramatical
José corre

José corrió

José corría

José estaba corriendo

Vendler (1967) fue uno de los primeros en incorporar estas nociones en una categorización de cuatro clases aspectuales del verbo: logros, realizaciones, actividades y estados. Se podrían añadir otros tres rasgos o parámetros semánticos (momentáneo, delimitado y dinámico) para distinguir entre sí estas clases aspectuales (Andersen y Shirai 1995; Bosque y Demonte 1999): momentáneo [+/-], que denota si un evento es puntual o si se da durante un período cuantificable; delimitado [+/-] que marca si el evento tiene una culminación temporal lógica e identificable; y dinámico [+/-] que se refiere a la progresión en el tiempo y al cambio del evento (tabla 2).

Tabla 2. Rasgos semánticos referidos al aspecto

\begin{tabular}{lcccc}
\hline Rasgos & Estado & Actividad & Realización & Logro \\
Momentáneo & - & - & - & + \\
Delimitado & - & - & + & + \\
Dinámico & - & + & + & + \\
\hline
\end{tabular}

Bajo este análisis de rasgos (tabla 3), los verbos de estado (EST), como querer, se diferencian de las otras tres categorías por su cualidad de no ser dinámicos [-dinámico]. Las actividades (ACT), las realizaciones (REA) y los logros (LOG), por otro lado, son [+ dinámico] y, por lo tanto, solo se pueden diferenciar por los rasgos [+/-] momentáneo y [+/-] delimitado. Los logros y las realizaciones son [+ delimitado], mientras que las actividades son [-delimitado] y no tienen una terminación de tiempo identificable. Las actividades y las realizaciones son [-momentáneo], mientras que los logros son [+momentáneo] y capturan el comienzo o terminación de un evento. 
Tabla 3. Categorización de clase aspectual y rasgos semánticos

\begin{tabular}{|l|l|}
\hline \multicolumn{1}{|c|}{ Clase aspectual } & \multicolumn{1}{c|}{ Rasgos semánticos } \\
\hline $\begin{array}{l}\text { Estado (EST) } \\
\text { Persiste sobre el tiempo sin necesidad de } \\
\text { energía física }\end{array}$ & $\begin{array}{l}\text { Momentáneo (+/-) } \\
\text { iOcurre en un momento } \\
\text { del tiempo específico? }\end{array}$ \\
\hline $\begin{array}{l}\text { Actividad (ACT) } \\
\text { Duración inherente sin una finalidad } \\
\text { específica }\end{array}$ & $\begin{array}{l}\text { Delimitado (+/-) } \\
\text { iTiene una finalidad de } \\
\text { tiempo específico? }\end{array}$ \\
\hline $\begin{array}{l}\text { Realización (REA) } \\
\text { Incluye la finalidad y duración inherente }\end{array}$ & $\begin{array}{l}\text { Dinámico (+/-) } \\
\text { ¿Demuestra una acción o } \\
\text { estado? }\end{array}$ \\
\hline $\begin{array}{l}\text { Logro (LOG) } \\
\text { Captura el comienzo o final de un evento }\end{array}$ \\
\hline
\end{tabular}

\subsection{EL PRESENTE PROGRESIVO EN ESPAÑOL}

En español, ambas formas, la sintética (presente simple) y la analítica (presente progresivo), pueden captar la noción semántica de "acción en curso" (Comrie 1976; Gili-Gaya 1964). Esto está ejemplificado en (2), un caso que fue tomado de la encuesta (véase la sección de metodología). De hecho, parece existir una opción entre estas formas en cuanto a expresar la noción semántica de "acción en curso" en el español.

(2) a. Sintética: "Construyen nuevos edificios cerca del centro comercial".

b. Analítica: "Están construyendo nuevos edificios cerca del centro comercial".

Comrie (1976) afirma que la forma analítica puede reemplazarse con la forma sintética sin perder la noción de "acción en progreso". Westfall (1995), por su parte, argumenta que estas formas son compatibles y que la duración de una no es menos que la otra y que, además, son semánticamente idénticas. Sin embargo, autores como Koontz-Garboden (2004: 1294) afirman que, aunque las dos formas en español coinciden en su significado, dado que ambas pueden marcar el aspecto progresivo, no se solapan en cuanto a la noción de verdad. Este autor afirma: "la interpretación del progresivo tiende a ser la única interpretación para la forma analítica mientras que la forma sintética añade una interpretación que expresa la habitualidad (frecuentativa) o repetición de un evento o acción”. Además, Koontz-Garboden (2004), argumenta que la forma analítica es favorecida en contextos donde podría surgir una ambigüedad de significado por el uso de la forma sintética. 
Koontz-Garboden (2004) ilustra su argumento con el ejemplo en (3) para respaldar su justificación de que la forma analítica es desfavorecida en los contextos con significado habitual (o frecuentativo). La tabla 4 resume el argumento de Koontz-Garboden sobre las diferencias de las formas sintéticas y analíticas y su uso en el presente progresivo en español.

(3) a. Como es joven, Miguel juega fútbol los domingos.

b. *Como es joven, Miguel está jugando fútbol los domingos.

Tabla 4. Resumen de las distinciones entre las formas analítica y sintética según Koontz-Garboden (2004)

\begin{tabular}{lcc}
\hline Criterio & Sintética & Analítica \\
\hline Esperado en contextos con significado & Sí & No \\
habitual & & \\
Interpretación progresiva solamente & No & Sí \\
Progresivo y habitual & Sí & No \\
\hline
\end{tabular}

Se puede ver en esta tabla que el autor predice un uso mayor de la forma sintética tanto en los contextos de significado habitual como en los contextos que combinan un significado habitual y de "acción en curso" (progresivo). Estas predicciones serán estudiadas con detenimiento en el análisis que se presenta en la sección de resultados.

Sin embargo, algunos autores, como Quesada (1995) y Torres Cacoullos (2000), afirman que esta caracterización se encuentra en variación en el mundo hispanohablante y que la forma analítica se encuentra más a menudo en los contextos frecuentativos. Quesada (1995) hace un análisis histórico de la forma del presente progresivo en español y afirma que la forma analítica se extiende a los siguientes contextos: a) actualización de la acción en el mismo momento en que se habla (están jugando al parchís), b) contemporaneidad, en la que la acción se extiende hacia el pasado y hacia el futuro (está estudiando Medicina), c) iteratividad o frecuencia (está viendo la televisión todos los días) que se acerca a la acción habitual, normalmente expresada mediante el presente simple. Nuestro estudio toma en cuenta estas consideraciones e investiga si una forma se favorece en los contextos codificados como frecuentativos y de inmediatez.

\subsection{MARCo TeÓRICO}

Varias investigaciones han demostrado que hay una correlación entre la distribución de la morfología y el aspecto-léxico semántico inherente 
al verbo (Bosque y Demonte 1999). Esta noción es analizada en algunos estudios pioneros llevados a cabo por Andersen $(1986,1991)$ y Anderson y Shirai $(1994,1995)$ quienes denominan el fenómeno como "la primacía del aspecto". Esta hipótesis sostiene que los hablantes son guiados por el aspecto semántico inherente al verbo en el momento de seleccionar una $u$ otra forma morfológica.

Una premisa importante de la hipótesis, "la predisposición de la distribución" (Andersen 1991, 1993; Andersen y Shirai 1994), predice que la forma progresiva aparecerá más con verbos durativos que con los verbos no durativos. Otra premisa, "el principio de la congruencia", señala que hay una conexión creciente cuando el significado de un morfema se acerca al significado del ítem léxico con el que se relaciona. Esto podría explicar por qué en inglés los verbos de actividad se asocian frecuentemente con marcadores de progresivo. En inglés, -ing tiene una conexión fuerte con los verbos de actividad dado que estos contienen los rasgos semánticos (+ dinámico, -momentáneo) y, además, es la única manera de marcar "acción en progreso" en esta lengua (Bybee et al. 1994). El presente estudio considera las afirmaciones de Andersen y Shirai (1994) y se basa en las clases aspectuales del verbo de Vendler (1967) para interpretar la distribución de las formas analíticas y sintéticas del presente progresivo en español y, a la vez, tratar la variación de estas formas.

\subsection{ESTUDIOS PREVIOS}

Klein (1980) comparó 8 informantes monolingües con 11 informantes bilingües del español y el inglés, los cuales vivían en Nueva York. La autora propone la transferencia (indirecta) como el factor principal para justificar la diferencia encontrada entre la selección de la forma analítica y sintética de estos participantes. Los resultados encontrados indicaron una disminución de la forma sintética por parte de los bilingües para expresar acción en curso (monolingües: 26\% sintética, $74 \%$ analítica; bilingües: $6 \%$ sintética, $94 \%$ analítica).

Klein concluye que los resultados comprueban su hipótesis de que los monolingües utilizarían la forma sintética más que los bilingües, quienes prefirieron la forma analítica. Esta autora señala que los bilingües favorecen (inconscientemente) la forma analítica más que los monolingües dado que la forma analítica empareja su sistema del español (L1) con el sistema del inglés (L2) sin afectar la gramaticalidad de las normas del español. Además, Klein apunta al deseo por parte de los bilingües de dominar el inglés, la lengua de prestigio en la comunidad neoyorquina, como otro factor que influye 
en el uso de la forma analítica por parte de los bilingües. Sus resultados muestran que hay variación entre la forma analítica y sintética en español y que los bilingües muestran una tendencia a utilizar la forma analítica más que los monolingües en los contextos de "acción en curso". No obstante, el estudio no indica la razón por la cual los monolingües en un inicio, antes de estar en contacto con el inglés, prefieren la forma sintética. También es importante señalar que el estudio compara el uso de la forma sintética frente a la analítica en contextos que no son idénticos.

Otro estudio que concuerda con los resultados de Klein (1980) es el de Koontz-Garboden (2004), quien reanaliza los datos de Klein bajo la Teoría de la Optimidad. El estudio se centra en factores semánticos y gramaticales más allá del factor extralingüístico de contacto para explicar por qué los bilingües prefieren la forma analítica en los contextos de acción en curso. Koontz-Garboden concluye que los bilingües prefieren la forma analítica no por motivos sociales como señala Klein, sino porque la congruencia de los sistemas gramaticales facilita la transferencia de estas formas. De igual importancia para los propósitos de nuestro estudio, es el estudio de CortésTorres (2005), quien examinó los factores lingüísticos y extralingüísticos y su efecto en el uso de las dos formas en el habla monolingüe y bilingüe del español puertorriqueño. La autora afirma que el aspecto léxico-semántico del verbo es un indicador estadísticamente relevante en la selección de las formas analíticas y sintéticas. Más revelador aún es que su estudio presenta evidencia en contra de investigaciones previas en las que se proponía la transferencia del inglés como factor principal en la selección de una u otra forma. Cortés-Torres muestra que ambos grupos en su estudio, tanto los bilingües como los monolingües, usaron las formas analíticas y sintéticas en los contextos codificados como "acción en curso": la forma analítica (24\%) y la forma sintética (76\%), respectivamente. Es decir, que en su estudio el bilingüismo como factor era mucho menos importante que otras variables, como el aspecto léxico-semántico del verbo.

En un estudio más reciente, Márquez Martínez (2009) analiza la variación de las formas analíticas y sintéticas en un grupo de hablantes puertorriqueños residentes en los EE.UU. Los hallazgos del análisis indican que el aspecto léxico-semántico del verbo, el valor aspectual de la oración y la variable extralingüística "sexo" son factores que contribuyen en la explicación del fenómeno. Específicamente, Márquez Martínez (2009: 120) señala que: "los factores que favorecen la perífrasis son: actividad-consecución (dentro del aspecto léxico-semántico del verbo); el aspecto durativo, progresivo o de duración limitada (dentro del aspecto de la oración) y el sexo masculino".

El presente estudio contribuye en la investigación de los factores lingüísticos y extralingüísticos que explican la variación entre las formas 
analíticas y sintéticas en dos grupos de hablantes, tanto monolingües como bilingües del español, a través de una encuesta contextualizada. El instrumento utilizado aquí fue previamente codificado según contextos lingüísticos. La metodología de la encuesta contextualizada pretende subsanar la falta de consistencia en los estudios anteriores en relación con la definición del contexto variable y el efecto de las variables lingüísticas. Este tipo de instrumento es importante porque provee una manera de analizar y controlar las variables con mayor precisión, lo cual nos permite medir el peso de las variables con más confiabilidad porque limita otros factores posibles que intervienen en el habla espontánea.

El presente estudio está guiado por las siguientes preguntas de investigación:

1. ¿Se encuentra variación de las formas analíticas y sintéticas en contextos idénticos de "acción en curso" en español o en los contextos frecuentativos? ¿podemos predecir la selección de una u otra forma basándonos en los factores lingüísticos y extralingüísticos?

2. ¿Hasta qué punto es el bilingüismo un factor relevante en la selección de la forma analítica o sintética? ¿Cómo se comparan nuestros resultados con los de estudios previos que proponen la transferencia como razón principal para la variación entre monolingües y bilingües?

\section{LA VARIACIÓN MORFOSINTÁCTICA DE LAS FORMAS ANALÍTICAS Y SINTÉTICAS}

\subsection{Metodología}

Por medio de una encuesta contextualizada y codificada según la clasificación semántica de los tipos de verbos y el valor semántico de los adverbios, examinamos los contextos en los que se usa la forma sintética y analítica en variación y el efecto que tienen los factores lingüísticos en dicha variación. Además de proveer un análisis cuantitativo de los factores lingüísticos, también se consideran los factores extralingüísticos de género y bilingüismo. Las encuestas fueron completadas por un total de 10 bilingües y 10 monolingües. El grupo de participantes monolingües lo forman hablantes de la Argentina, Perú, Venezuela y México, entre los 25 y 50 años, quienes nunca han vivido en un país anglófono. El grupo de participantes bilingües lo forman hablantes de Perú, México, Argentina, 
Colombia, España, y Costa Rica, entre los 26 y 44 años, quienes viven y trabajan en los Estados Unidos. Estos participantes bilingües completaron la escuela secundaria en un ambiente monolingüe en español, y se consideran bilingües por haber vivido un promedio de 9,5 años en los Estados Unidos. En ambos grupos se ve una representación de diversas regiones dialectales. Casi todos los participantes tanto los monolingües como bilingües tienen un nivel de educación de postgrado y son de la clase media o media-alta. Este último hecho es importante, dado que los factores que consideran el nivel de educación y clase social del participante podrían influir en la selección de estas formas (Torres Cacoullos 2000).

Dada la complejidad de los factores que intervienen en los estudios de aspecto-tiempo (Bardovi-Harlig, 2000) utilizamos una encuesta contextualizada para controlar los rasgos lingüísticos y, a la vez, proveer contextos idénticos para poder comparar los usos. En algunos estudios sobre la cópula y el subjuntivo en el español, Geeslin (2003) y Gudmestad (2006), se emplea un instrumento parecido y sus resultados nos indican que el instrumento podría ser eficaz para los propósitos de nuestra investigación.

La encuesta consistía en 19 oraciones en total y se incluyeron 3 preguntas para distraer la atención de los participantes en relación con el fenómeno lingüístico estudiado aquí. Las 16 oraciones fueron codificadas según la semántica verbal (estados, actividades, logros, realizaciones) y por el valor semántico del adverbio, de frecuencia (todos los días, cada día) y de inmediatez (ahora, en este momento), para investigar la influencia que tienen estos factores en la selección de una u otra forma del presente progresivo: la analítica o el sintético.

La encuesta contextualizada fue codificada para tomar en cuenta los siguientes factores:

\section{1.) Aspecto léxico-semántico del verbo}

a) verbos de actividad: correr, tocar (la guitarra), caminar, jugar (fútbol)

b) logros: salir, empezar, llegar

c) realizaciones: comer algunas galletas, caminar $5 \mathrm{~km}$, construir edificios

d) estados: creer, necesitar, saber, querer

\section{2.) Valor semántico del adverbio}

a) Inmediatez: ahora, en este momento. Estos añaden un valor de inmediatez al predicado/contexto y no se extiende a más tarde o mañana por ejemplo. 
b) Frecuencia/acción repetitiva: todos los días, cada día. Estos indican que el predicado/contexto ocurre con regularidad.

\section{3.) Factores extralingüísticos}

a) El género: masculino o femenino.

b) Bilingüismo: monolingüe del español o bilingüe del español-inglés.

Para el análisis co-variacional de los datos se empleó el programa estadístico GoldVarb X, que distingue los factores lingüísticos y extralingüísticos que influyen en la realización de una u otra variante (Sankoff, Tagliamonte y Smith 2005). Dentro de este programa, los grupos de factores que se utilizaron para codificar los datos fueron los siguientes:

\section{VARIABLE DEPENDIENTE \\ a- Verbo en forma analítica \\ s- Verbo en forma sintética \\ d- Ambas formas}

\section{VARIABLES INDEPENDIENTES}

Grupo de factores 1: Aspecto léxico-semántico del verbo

e- estados

a- actividad

r- realización

1- logro

\section{Grupo de factores 2: Valor semántico del adverbio}

i- inmediatez

f- habitual o frecuentativo

s- sin adverbio de este tipo

\section{Grupo de factores 3: Género}

f- femenino

m- masculino

\section{Grupo de factores 4: EI bilingüismo}

$\mathrm{m}$ - monolingüe

b- bilingüe

Primero, todos los participantes llenaron un cuestionario sobre su experiencia previa con el inglés, el español y sobre otros datos personales como su edad, región, lugar de nacimiento y género. Después, los participantes 
llenaron la encuesta sin provisiones de tiempo. Las instrucciones les pedían que marcaran con una $\mathrm{X}$ una de las opciones para indicar qué dirían en cada situación. Como se nota no se les pidió nada sobre la gramaticalidad, sino se les dio la libertad de mostrar su preferencia (indirectamente) hacia una de las dos formas, la analítica o sintética, en cada contexto, o una preferencia hacia ambas.

\section{Ejemplo tomado de la encuesta:}

Juan le pregunta a Víctor dónde se encuentra su hermano en este momento. Víctor le responde:

A. "Camina en el parque ahora".

B. "Está caminando en el parque ahora". Prefiero A. Prefiero B. Ambos.

Codificación: [+Actividad: caminar] [+ Inmediatez: ahora]

\section{RESULTADOS Y DISCUSIÓN}

Hemos organizado los resultados con el objeto de dar respuesta a las preguntas de investigación propuestas previamente. En primer lugar, nos ocuparemos de determinar el efecto de los factores lingüísticos en los contextos en los que los hablantes coinciden en aceptar el uso variable de ambas formas. De igual forma, observamos el efecto del factor bilingüismo en la predicción de los contextos variables. Por lo tanto, el valor de aplicación elegido para el análisis estadístico consiste en la observación de aquellos contextos donde los participantes consideraron aceptable el uso tanto de la forma analítica como de la forma sintética. El cuadro 1 muestra la distribución de los datos en la muestra analizada.

Cuadro 1. Aceptabilidad de las formas verbales según los contextos propuestos

\begin{tabular}{lcc}
\hline Aceptabilidad & Número de casos & Porcentaje \\
Aceptabilidad de ambas formas & $62 / 320$ & $19.4 \%$ \\
Aceptabilidad de la forma analítica & $78 / 320$ & $24.4 \%$ \\
Aceptabilidad de la forma sintética & $180 / 320$ & $56.2 \%$ \\
\hline
\end{tabular}


El cuadro 1 revela que el contexto variable alcanza un 19.4\%. En el análisis de los datos que presentamos a continuación nos enfocamos en identificar las variables que contribuyen a explicar el ámbito de la variación en este contexto. El cuadro 2 presenta los resultados del análisis multivariado realizados mediante el uso del programa VARBRUL en su versión GoldVarb. Este programa ha sido especialmente diseñado para el estudio de la variación lingüística. Específicamente, los datos fueron analizados empleando la regresión logística la cual permite determinar qué conjunto de factores contribuye en la selección del valor de aplicación, el cual ha sido definido como la opción donde los hablantes consideraron aceptable el uso de ambas formas. Hay que destacar que la prueba no les preguntaba nada sobre la gramaticalidad.

Como se ve en el cuadro 2, los factores lingüísticos identificados como significativos por el programa GoldVarb son el aspecto léxico-semántico del verbo y el valor semántico del adverbio. Al mismo tiempo, el programa identifica el factor extralingüístico del bilingüismo como una variable que favorece la alternancia de las formas analíticas y sintéticas en el español. Vale destacar que un número considerable de los contextos produjo respuestas en las que ambas formas eran aceptables, lo que confirma que existe variación entre estas formas.

Cuadro 2. Grupos de factores seleccionados por GoldVarb como significativos para la aceptabilidad de ambas formas: la analítica o la sintética (medio-corregido $=0.131 ; \mathrm{p} \leq 0.025 ; \log$ likelihood $=-131.739$ )

\begin{tabular}{|l|c|c|c|c|}
\hline Grupos de factores & Factores & Peso & $\mathbf{\%}$ & N \\
\hline \multirow{3}{*}{$\begin{array}{l}\text { Aspecto léxico-se- } \\
\text { mántico del verbo } \\
\text { Rango }=607\end{array}$} & Logros & 0.724 & 30.0 & $24 / 80$ \\
\cline { 2 - 5 } & Realizaciones & 0.665 & 25.0 & $20 / 80$ \\
\cline { 2 - 5 } & Actividades & 0.591 & 20.0 & $16 / 80$ \\
\cline { 2 - 5 } & Estados & 0.117 & 2.5 & $2 / 80$ \\
\hline \multirow{2}{*}{$\begin{array}{l}\text { Valor semántico del } \\
\text { adverbio }\end{array}$} & Frecuentativo & 0.732 & 35.0 & $28 / 80$ \\
\cline { 2 - 5 } Rango = 337 & Inmediatez & 0.461 & 16.2 & $13 / 80$ \\
\hline \multirow{2}{*}{$\begin{array}{l}\text { El bilingüismo } \\
\text { Rango }=186\end{array}$} & Ninguno & 0.395 & 13.1 & $21 / 160$ \\
\cline { 2 - 5 } & Bilingüe & 0.593 & 24.4 & $39 / 160$ \\
\hline
\end{tabular}

Comenzaremos nuestro análisis con el grupo de factores referente al aspecto léxico-semántico del verbo. Los verbos de logros (0.724), realizaciones $(0.665)$ y de actividades $(0.591)$ favorecen la posibilidad 
de elegir ambas formas, mientras que los verbos de estados (0.177) la desfavorecen. El hecho de que los estados desfavorecen la posibilidad de ambas formas es lógico, considerando que estos tienen una tendencia de aparecer casi únicamente con la forma sintética $(96.2 \%$ en nuestros resultados). Por otro lado, observamos que los participantes aceptan tanto una como otra forma con los verbos de logros, realizaciones y de actividades en un número de contextos que es mayor según la comparación de nuestros datos con estudios anteriores. En principio, es importante señalar que los verbos que tienen menos probabilidad de aparecer en variación entre las formas analíticas y sintéticas son los verbos de actividades y estados. Esto se explica porque estos verbos tienen más probabilidad de aparecer con una sola forma: la sintética en el caso de los verbos de estados y la analítica en el caso de los verbos de actividades. Sin embargo, el descubrimiento de que los verbos de logros y realizaciones favorecen la variación y, sobre todo, que los verbos de logros no favorecen la forma sintética, requiere una explicación mayor. Consideramos que el contexto que proveen los verbos de logros y realizaciones es ideal para hablar de la variación, dado que los verbos de actividades ya son terreno preferentemente de la forma analítica y los estados, de la forma sintética. En otras palabras, es justamente en estos contextos donde se requiere un análisis mayor de los factores que influyen en la selección de una u otra forma, debido a que el factor léxico-semántico del verbo en sí no puede predecir el uso de la forma analítica o sintética.

Los verbos de logros y realizaciones comparten el rasgo [+] delimitado, que implica una culminación temporal identificable. De igual manera, las realizaciones comparten tanto los rasgos semánticos de las actividades [+ dinámico], como los de los verbos de estados [-momentáneo]. Dadas estas consideraciones, interpretamos la aceptabilidad de ambas formas como un reconocimiento por parte del hablante de que el uso de una u otra forma (presente simple o presente progresivo) en estos contextos acentuaría uno de estos rasgos semánticos. En otros términos, esta variación se puede interpretar como una estrategia discursiva de los hablantes para manipular el aspecto gramatical de la oración y diferenciarlo del aspecto inherente al verbo (Bardovi-Harlig 2000; Comrie 1976; Smith 1991).

En cuanto a los resultados sobre el valor semántico del adverbio, se observa que el valor semántico de frecuencia/acción repetitiva (todos los días, cada día aumenta la probabilidad de variación (0.732) mientras que el valor semántico de inmediatez no lo aumenta(0.461). Este hallazgo se explica en parte porque los contextos en que el adverbio añade un significado de inmediatez (ahora, en este momento) favorecen el uso de la forma analítica. Al mismo tiempo, estos resultados muestran la importancia de considerar el valor semántico de frecuencia/acción repetitiva en los estudios del presente 
progresivo y apoyan las suposiciones de Quesada (1995) y Torres Cacoullos (2000) de que tanto la forma analítica como la forma sintética aparecen en contextos con un significado frecuentativo. El hecho de que este contexto parece significativo para la variación puede mostrar que estas formas están en un proceso de gramaticalización en la lengua española actual (Márquez Martínez 2009; Torres Cacoullos 2000).

Finalmente, los resultados indican un aumento de uso de la forma analítica en el caso de los participantes bilingües. El hecho de que los hablantes bilingües escojan la forma analítica en más contextos que los monolingües parece concordar con los resultados de Klein (1980), quien adjudica tal incremento de uso para los bilingües a una influencia del inglés, lengua que les rodea y emplean los participantes de nuestro estudio de manera cotidiana en los Estados Unidos.

\section{CONCLUSIÓN}

Por medio de una encuesta contextualizada y controlada mediante la inclusión de variables lingüísticas y extralingüísticas, se demuestra que las formas sintéticas y analíticas aparecen en variación en un número de contextos mayor que los previamente identificados en otros estudios. Esto se debe en parte a la inconsistencia entre los contextos comparados en los estudios previos en los cuales la variación no había sido totalmente especificada. Aunque no ponemos en duda el valor de los datos obtenidos del habla espontánea, el tipo de instrumento empleado en nuestro estudio resultó ser eficaz dada su capacidad para proporcionar datos de todos los contextos posibles y presentarlos de una manera homogénea a los participantes.

Los resultados del estudio apuntan a que los factores lingüísticos del aspecto léxico-semántico del verbo y del valor semántico del adverbio, además del factor extralingüístico del bilingüismo, están altamente relacionados en cuanto a la selección de la forma analítica o la forma sintética en los contextos cuyo significado alude al presente progresivo del español. Por un lado, nuestros resultados concuerdan con algunos autores como Quesada (1995) o Márquez Martínez (2009) sobre un incremento de uso de la forma analítica en los contextos frecuentativos pero, por otro lado, otros hallazgos como el hecho de que los verbos de logros no favorecen la forma sintética va en contra de las predicciones de autores como Comrie (1976). En nuestros datos hemos identificado que el contexto de los verbos de 
logros y realizaciones favorece la variación, especialmente en los hablantes bilingües. Este patrón identificado parece ser novedoso en relación con los datos reportados en estudios previos. Se puede considerar que este es un resultado importante para los estudios que tratan la variación de las formas analíticas y sintéticas y es un tema que requiere ser investigado con mayor profundidad.

De igual importancia es el hecho de que nuestros resultados concuerden con los de Klein (1980) en que los bilingües (español-inglés) de nuestro estudio emplean un mayor uso de la forma analítica, pero que al mismo tiempo los participantes monolingües, sin estar en contacto con el inglés, utilizan esta forma en un número de casos mayor según se ha determinado en la literatura previa. Este último hecho se podría interpretar como evidencia a favor de Torres Cacoullos $(2000,2008)$ de que la forma analítica se extiende en el español monolingüe de hoy, sin el contacto con el inglés. Es posible suponer que el español monolingüe está en un proceso de cambio como apunta Torres Cacoullos (2000), pero que se acelera por el contacto con el inglés como se ha propuesto previamente (Silva-Corvalán 2001).

En adelante queda por llevar a cabo otros estudios de este tipo y ampliar el análisis para incorporar tanto un número de factores lingüísticos mayor, como la frecuencia léxica, los campos semánticos del verbo y varios aspectos temporales de la oración, así como incluir más factores extralingüísticos, tales como la clase social, el país de origen y la edad de los informantes, en un esfuerzo continuo de revelar cómo los datos lingüísticos y extralingüísticos intervienen en la selección de las formas analíticas y sintéticas que indican el presente progresivo en español.

\section{REFERENCIAS BIBLIOGRÁFICAS}

ANDERSEn, Roger W. 1986. El desarrollo de la morfología verbal en el español como segundo idioma. En J. M. Meisel (ed.)., Adquisición de lenguaje/Aquisição da linguagem, pp. 115-138. Frankfurt: Vervuert.

1991. Developmental sequences: The emergence of aspect marking in second language acquisition. En C. A. Ferguson y T. Huebner (eds.). Second language acquisition and linguistic theories, pp. 305-324. Amsterdam: Benjamins. 
1993. Four operating principles and input distribution as explanations for underdeveloped and mature phonological systems. En K. Hyltenstam y A. Viborg (eds.). Progression and regression in language, pp. 309-339. Cambridge, UK: Cambridge University Press.

Andersen, Roger W. y Yasuhiro Shirai. 1994. Discourse Motivations for Some Cognitive Acquisition Principles. Studies in Second Language Acquisition 16 (2): 133-156.

1995. TheAcquisition of Tense-Aspect Morphology: A Prototype Account. Language 71 (4): 743-762.

Bardovi-Harlig, KathleEn. 2000. Tense and aspect in second language acquisition: Form, meaning, and use. Malden, MA: Blackwell.

Bosque, Ignacio y Violeta Demonte. 1999. Gramática descriptiva de la lengua española, vol. II. Madrid: Espasa-Calpe.

Butt, John y Carmen Benjamin. 1994. A New Reference Grammar of Modern Spanish, $2^{\circ}$ edition. London y New York: Edward Arnold.

Bybee, Joan, Revere Perkins y William Pagliuca. 1994. The evolution of grammar: tense, aspect, and modality in the languages of the world. Chicago: University of Chicago Press.

Comrie, Bernard. 1976. Aspect. New York: Cambridge University Press.

Cortés-Torres, Mayra. 2005. ¿Qué estás haciendo?: la variación de la perífrasis estar + ndo en el español puertorriqueño. Selected Proceedings of the 7th Hispanic Linguistics Symposium. 42-55.

Geeslin, Kimberly L. 2003. A comparison of copula choice in advanced and NS Spanish. Language Learning 53.703-764.

2006. Linguistic contextual features and variation in L2 data elicitation. In C. Klee y T. Face (eds.). Selected proceedings from the 7th Conference on the acquisition of Spanish and Portuguese as a first and second language, pp. 74-85. Somerville, MA: Cascadilla Press.

Gili Gaya, Samuel. 1964. Curso superior de sintaxis española. Barcelona: Bibliógrafa, S.A.

Gudmestad, Aarnes. 2006. L2 Variation and the Spanish subjunctive: Linguistic features predicting mood selection. En C. Klee y T. Face (eds.), Selected proceedings from the $7^{\text {th }}$ CLASP, pp. 170-184. Somerville, MA: Cascadilla Press.

KLEIN, FlORA. 1980. A quantitative study of syntactic and pragmatic indications of change in the Spanish of bilinguals in the U.S. En William Labov (ed.). Locating Language in Time and Space, pp. 69-82. New York: Academic Press.

Koontz-Garboden, Andrew. 2004. Language contact and Spanish aspectual expression: a formal analysis. Lingua 114: 1291-1330.

Labov, William. 1972. Sociolinguistic Patterns. Philadelphia: University of Pennsylvania Press.

Márquez Martínez, Miguel Ángel. 2009. La perífrasis estar $+n d o$ en puertorriqueños bilingües con residencia en Estados Unidos. Boletín de Filología, Tomo XLIV, 2: 119134.

Mourelatos A. 1978. Events, processes, and states. Linguistics and Philosophy 2: 415434.

Quesada, J. Diego. 1995. Estar + ndo y el aspecto progresivo en español. Iberoromania 42: 8-29.

SÁncheZ-Muñoz, A. 2004. Transfer in the Spanish Progressive Constructions in Los Angeles. USC Working Papers in Linguistics 2: 16-29. 
Sankoff, David, Sali Tagliamonte y Eric Smith. 2005. GoldVarb X: a variable rule application for Macintosh and Windows. Department of Mathematics, University of Ottawa and Department of Linguistics, University of Toronto.

SANKofF, David. 1988. Sociolinguistics and syntactic variation. En Frederic Newmeyer (ed.). Linguistics: The Cambridge Survey. IV: The Socio-Cultural Context. Cambridge, MA: Cambridge University Press.

Silva-Corvalán, Carmen. 2001. Sociolingüística y pragmática del español. Washington, D.C.: Georgetown University Press.

Smith, Carlota S. 1991. The Parameter of Aspect. Dordrecht, The Netherlands: Kluwer Academic Publishers.

Torres Cacoullos, Rena. 2000. Grammaticization, Synchronic Variation, and Language Contact: A study of Spanish progressive -ndoconstructions. Amsterdan/Philadelphia: John Benjamins.

2008. La evolución de estar + V-ndo hacia expresión obligatoria del aspecto progresivo en español. Concepción Company Company y José G. Moreno de Alba (editores), Actas VII Congreso Internacional de Historia de la Lengua Española, pp. 1147-1162. Madrid: Arco Libros.

Vendler, Zeno. 1967. Linguistics in Philosophy. Ithaca, NY: Cornell University Press.

Westrall, Ruth. E. 1995. Simple and Progressive Forms of the Spanish Past Tense System: A Semantic and Pragmatic Study in Viewpoint Contrast. Ph. D. Thesis, University of Texas, Austin, Austin, TX. 